$\mathrm{DE}$

M E D I C I N A

T R O P I C A L

$\mathrm{DE}$

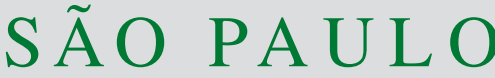

JOURNAL OF THE SÃO PAULO INSTITUTE OF TROPICAL MEDICINE

${ }^{1}$ Secretaria Municipal de Saúde, Santo André, São Paulo, Brazil

2Universidade do Estado de São Paulo, Campus Botucatu, Botucatu, São Paulo, Brazil

${ }^{3}$ Instituto Adolfo Lutz, Centro de Laboratório Regional Santo André, Santo André, São

Paulo, Brazil

${ }^{4}$ Instituto Adolfo Lutz, Centro de Virologia, São Paulo, São Paulo, Brazil

${ }^{5}$ Instituto Adolfo Lutz, Centro de Imunologia, São Paulo, São Paulo, Brazil

Correspondence to: Luís Fernando de Macedo Brígido Instituto Adolfo Lutz, Centro de Virologia, Av. Dr. Arnaldo, 355, CEP 05411001, São Paulo, SP, Brazil

Tel: +55 1130682982

E-mail: lubrigido@gmail.com

Received: 20 August 2021

Accepted: 8 November 2021

\section{SARS-CoV-2 testing among patients and healthcare professionals in an HIV outpatient clinic in Brazil}

\author{
Elaine Monteiro Matsuda1, Isabela Penteriche de Oliveira ${ }^{2}$, Ivana Barros de \\ Campos $^{(13}$, Cintia Mayumi Ahagon ${ }^{4}$, Marcia Jorge Castejon ${ }^{5}$, Valeria Oliveira \\ Silva ${ }^{4}$, Fernanda Matsuda Manzoni ${ }^{1}$, Giselle Ibette López-Lopes ${ }^{4}$, Luís \\ Fernando de Macedo Brígido ${ }^{\circledR} 4$
}

\section{ABSTRACT}

The COVID-19 pandemic in Brazil has been marked by high infection and death rates. The immune response generated by current vaccination might be influenced by previous natural infection, and baseline estimates may help in the evaluation of vaccine-induced serological response. We evaluated previous SARS-CoV-2 testing (RT-PCR), and performed rapid diagnostic tests (RDT) and high throughput electrochemiluminescence immunoassay (ECLIA) before vaccination among people living with HIV (PLWH), users of antiretroviral prophylaxis (PrEP/PEP), and healthcare professionals in an HIV outpatient clinic (HCP-HC). RDT was positive in $25.7 \%$ (95\% CI: $19-33 \%$ ) overall, $31.3 \%$ (95\% CI : 18-45\%) among PLWH, 23.7\% (95\% CI : 14-34\%) in PrEP/PEP users and $21.4 \%$ (95\% CI : $05-28 \%$ ) in HCP-HC ( $\mathrm{p}=0.548)$. Diagnostic RT-PCR testing was very limited, even for symptomatic individuals, and whereas all HCP-HC had one test perfomed, only $35 \%$ of the patients (PREP/ $\mathrm{PEP} / \mathrm{PLWH})$ were tested $(\mathrm{p}<0.0001)$. Adequate monitoring of post-vaccination humoral response and breakthrough infections including those in asymptomatic cases are warranted, especially in immunologically compromised individuals.

KEYWORDS: SARS-CoV-2. HIV. PrEP. PEP. Health care personals. Prevalence.

\section{INTRODUCTION}

Molecular and antigen detection tests are the key for COVID-19 diagnosis and allow the control of viral transmission. Viral detection is generally of short duration and serology may be a surrogate of previous infections, but titers may wane with time $^{1}$. Vaccination generally induces seroconversion, but the long-term persistence of these antibodies is still unknown. Moreover, both infected and vaccinated individuals may not have detectable antibodies in some assays ${ }^{2}$. Albeit its limitations, serology is a simple and valuable tool to guide public health policies, and pre-vaccination estimates may be useful to understand the impact of vaccination on specific antibody production. Brazil has been experiencing large infection rates with consequent high mortality due to the lack of adequate non-pharmacological measures, disinformation from authorities associated with an initially slow pace of vaccination. As of October $8^{\text {th }}, 2021$, a total of 235,087,603 vaccine doses had been administered and 21,516,967 confirmed cases of COVID-19 resulting in 599,359 deaths had been reported to $\mathrm{WHO}^{3}$. Given that more than half of Brazilians will be completely vaccinated by the end of 2021, we are now experiencing the first real drop in the number of deaths due to COVID-19 ${ }^{3}$. This new phase, with COVID-19 evolving 
in a predominant vaccinated population, may pose new questions. Baseline information may be a key instrument to understand the evolution of infection in this post vaccination era. COVID-19 has been associated with more severe diseases in people living with HIV (PLWH $)^{4}$ and the immunodeficiency may hamper vaccines' responses. To assess pre-vaccination COVID-19 serology, we evaluated patients seeking HIV pharmacological prophylaxis, patients living with HIV and healthcare professionals in an HIV outpatient clinic.

\section{MATERIALS AND METHODS}

We conducted a cross-sectional study among patients living with HIV (PLWH), users of pre (PrEP) or post exposure (PEP) pharmacological prophylaxis to assess prior exposures to SARS-CoV-2. Blood samples were collected two months before the beginning of COVID-19 vaccination, that is, at the end of the pre-vaccination period. $\mathrm{HCP}$ of this unit (HCP-HC), an HIV outpatient clinic located in Santo Andre city $\left(23.6789^{\circ} \mathrm{S}, 46.5367^{\circ} \mathrm{W}\right)$ that is not a COVID-19 reference center, in the metropolitan area of Sao Paulo State was also tested in the same period to compare the prevalences of positive serological tests. The study was offered to all HCW users, taking advantage of the time of HIV testing (PEP/PrEP users) or HIV monitoring testing (HIV patients). All HCP-HC accepted to participate in the study, and less than $5 \%$ of invited patients refused the enrolment.

All volunteers had plasma and serum samples collected and submitted to a Rapid Diagnostic Test (RDT, Wondfo ${ }^{\oplus}$ 2019-nCoV Antibody Test), a qualitative lateral flow immunochromatographic assay (Guangzhou Biotech Co. Ltd., China) that detects specific IgG/IgM antibodies to SARS-CoV-2 by binding with the domain of the viral spike protein (S); most participants were also submitted to an IgG antibody test to SARS-CoV-2 nucleocapsid protein (NP) antigen (Electrochemiluminescence immunoassay ECLIA, Elecsys ${ }^{\circledR}$, Roche, Switzerland).

The study was approved by the institutional ethical committee (CAAE: 21164819.7.0000.0082) and all participants provided their informed consent. A questionnaire was applied to obtain information on previous symptoms compatible with COVID-195 ${ }^{5}$, as fever or chills, cough, shortness of breathe or difficulty breathing, fatigue, muscle or body aches, headache, loss of taste or smell, sore throat, congestion or runny nose, nausea, vomiting, diarrhea, previous serological tests and SARS-CoV-2 RNA detection by RT-PCR (reverse transcriptase-polymerase chain reaction) in nasopharyngeal secretions.

Continuous variables were presented as median and interquartile range (IQR), and categorical variables as proportions. Confidence Intervals of $95 \%$ (95\% CI) were calculated using a binomial exact test. The Kruskal-Wallis test was used for continuous variables and Pearson's chisquare test or two-tailed Fisher Exact test as appropriate, to compare categorical variables using the software Stata 14 (StataCorp, College Station, TX, USA).

\section{RESULTS}

From November 2020 to January 2021, the study enrolled 152 consecutive volunteers; $31.6 \%$ were PLWH, 50\% PrEP/ PEP and $18.4 \%$ HCP-Cl. Table 1 shows demographic, clinical and laboratory data according to the inclusion group. Only 51\% (29/57) of all volunteers who reported previous COVID-19-related symptoms had an RT-PCR diagnostic test performed, usually 3-7 days after the onset of symptoms. . All patients' categories (PLWH/PrEP/PEP) showed lower rates of molecular testing $(35 \%, 15 / 43)$, as compared to HCP-HC $(14 / 14, \mathrm{p}<0.0001)$. All volunteers performed a serological RDT test, that was positive in $25.7 \%$ (95\% CI : 19-33\%), with 31.3\% (95\% CI: $18-45 \%$ ) positivity among PLWH, $23.7 \%$ (95\% CI: 14-34\%) among PrEP/PEP users and 21.4\% (95\% CI: 05-28\%) for HCP-HC $(\mathrm{p}=0.548)$. Rates varied among groups (Table 1), but the difference was not significant. As expected, rates were higher among previous symptomatic cases (23/57 or $40.4 \%$ ) and those with detected viral RNA (14/20 or $70 \%)$. The results of RDT serology were in accordance with ECLIA in 97 (84\%) of 116 participants tested by both assays, 10 reactive RDT and nine non-reactive RDT with ECLIA discordant results, providing similar prevalence estimates. For those previously tested for viral RNA by RT-PCR, serology was performed within a median of 183 days (IQR 59-272) after COVID-19 diagnosis (RNA detection by RT-PCR). Negative serology was documented in $19 \%$ by ECLIA and $30 \%$ by RDT. A previous serological test was referred by 53 participants, with six (9.6\%) positive results. Two out of seven cases referring previous positive serology were negative by RDT.

\section{DISCUSSION}

During the first year of the pandemic, the lack of vaccines associated with an awkward response that promoted chemical prophylaxis and disregarded the use of face masks and other non-pharmacological measures (albeit being clearly recommended by the scientific community), characterized the Brazilian response to COVID-19. Moreover, SARS-CoV-2 (RT-PCR or viral antigen) testing, correct diagnoses and contacts tracing 
Table 1 - Demographic, clinical and laboratory characteristics of volunteers.

\begin{tabular}{|c|c|c|c|c|c|c|}
\hline & ALL & $\mathrm{PLWH}^{1}$ & PrEP$^{2}$ & PEP $^{3}$ & $\mathrm{HCP}-\mathrm{HC}^{4}$ & $\mathrm{p}$ \\
\hline $\mathrm{N}(\%)$ & 152 & $48(31.6 \%)$ & $40(26.3 \%)$ & $36(23.7 \%)$ & $28(18.4 \%)$ & \\
\hline \multirow{2}{*}{$\begin{array}{l}\text { Gender } \\
\text { male }\end{array}$} & 110/152 (72.36\%) & $37 / 48$ (77.08\%) & $39 / 40(97.50 \%)$ & $29 / 36$ (80.56\%) & 05/28 (17.85\%) & 0.001 \\
\hline & $95 \% \mathrm{Cl}: 65-80$ & $95 \% \mathrm{Cl}: 65-89$ & $95 \% \mathrm{Cl}: 92-100$ & $95 \%$ Cl: 67-94 & $95 \% \mathrm{Cl}: 3-33$ & \\
\hline \multirow{2}{*}{ Age (years) } & 35 & 42.5 & 33.5 & 27 & 40 & 0.001 \\
\hline & IQR 27-46 & IQR 32-53 & IQR 29-40 & IQR 21-37 & IQR 30-52 & \\
\hline \multirow{2}{*}{$\begin{array}{l}\text { Ethnicity } \\
\text { Caucasian }\end{array}$} & 87/152 (57.23\%) & $23 / 48(47.91 \%)$ & $20 / 40(50 \%)$ & $20 / 36(55.56 \%)$ & $24 / 28(85.71 \%)$ & 0.008 \\
\hline & $95 \%$ Cl: 49-65 & $95 \%$ Cl: 33-63 & $95 \%$ Cl: 34-66 & $95 \% \mathrm{Cl}: 39-73$ & $95 \% \mathrm{Cl}: 72-100$ & \\
\hline \multirow{2}{*}{$\mathrm{HCP}^{5}$} & $45 / 148(30.40 \%)$ & $05 / 47(10.64 \%)$ & 06/39 (15.38\%) & 06/34 (17.65\%) & $28 / 28(100 \%)$ & $0.648^{*}$ \\
\hline & $95 \% \mathrm{Cl}: 23-38$ & $95 \% \mathrm{Cl}: 1-20$ & $95 \% \mathrm{Cl}: 4-27$ & $95 \% \mathrm{Cl}: 4-31$ & & \\
\hline \multirow{2}{*}{$\begin{array}{l}\text { Suggestive } \\
\text { symptoms }\end{array}$} & $55 / 148(38.51 \%)$ & $19 / 47$ (40.42\%) & 13/39 (33.33\%) & $11 / 34(32.35 \%)$ & $14 / 28(50 \%)$ & 0.454 \\
\hline & $95 \% \mathrm{Cl}: 31-46$ & $95 \%$ Cl: $26-55$ & $95 \%$ Cl: $18-49$ & $95 \%$ Cl: $16-49$ & $95 \%$ Cl: $30-70$ & \\
\hline \multirow{2}{*}{$\begin{array}{l}\text { RT-PCR } \\
\text { performed }\end{array}$} & $42 / 152(28.37 \%)$ & $09 / 47$ (19.14\%) & $03 / 39$ (7.69\%) & 13/34 (38.23\%) & $17 / 28(60.71 \%)$ & 0.001 \\
\hline & $95 \% \mathrm{Cl}: 21-36$ & $95 \% \mathrm{Cl}: 7-31$ & $95 \% \mathrm{Cl}: 1-16$ & $95 \%$ Cl: $21-55$ & $95 \% \mathrm{Cl}: 41-80$ & \\
\hline \multirow{2}{*}{$\begin{array}{l}\text { RT-PCR } \\
\text { detected }\end{array}$} & $20 / 42(47.61 \%)$ & 07/09 (77.78\%) & $02 / 03(66.67 \%)$ & $08 / 13(61.54 \%)$ & $03 / 17(17.65 \%)$ & 0.013 \\
\hline & $95 \% \mathrm{Cl}: 32-63$ & $95 \% \mathrm{Cl}: 44-100$ & $95 \%$ Cl: 0-100 & 95\% Cl: 31-92 & $95 \% \mathrm{Cl}: 0-38$ & \\
\hline \multirow{2}{*}{$\mathrm{RDT}^{6}$ reactive } & $39 / 152(25.66 \%)$ & $15 / 48(31.25 \%)$ & $05 / 40(12.50 \%)$ & $13 / 36(36.11 \%)$ & $06 / 28(21.42 \%)$ & 0.081 \\
\hline & $95 \%$ Cl: $19-33$ & $95 \%$ Cl: $18-45$ & $95 \% \mathrm{Cl}: 2-23$ & $95 \% \mathrm{Cl}: 20-53$ & $95 \% \mathrm{Cl}: 5-38$ & \\
\hline \multirow{2}{*}{ ECLIA $A^{7}$ reactive } & $31 / 116(26.72 \%)$ & $11 / 41(26.83 \%)$ & $07 / 34(20.60 \%)$ & 10/27 (37.04\%) & 03/14 (21.42\%) & 0.509 \\
\hline & $95 \% \mathrm{Cl}: 19-35$ & $95 \% \mathrm{Cl}: 13-41$ & $95 \% \mathrm{Cl}: 6-35$ & $95 \%$ Cl: $18-57$ & $95 \% \mathrm{Cl}: 0-46$ & \\
\hline
\end{tabular}

$\mathrm{PLWH}^{1}=$ people living with HIV; $\mathrm{PrEP}^{2}=$ pre-exposure prophylaxis; $\mathrm{PEP}^{3}=$ post-exposure prophylaxis; $\mathrm{HCP}^{-\mathrm{HC}^{4}=}$ health care personal in a HIV outpatient clinic; $\mathrm{HCP}^{5}$ = health care personal; $\mathrm{RDT}^{6}$ = rapid diagnostic test Wondfo ${ }^{\circledR}$ SARS-CoV-2; $\mathrm{ECLIA}^{7}=$ electrochemiluminescence immunoassay Elecsys ${ }^{\circledR}$ Anti-SARS-CoV-2. Age was presented as median and interquartile range IQR, and compared using Kruskal-Wallis test. Categorical variables were presented as proportions, and compared using Pearson's chisquared test. The $\mathrm{HCP}-\mathrm{HC}^{4}$ group, all by definition $\mathrm{HCP}$, are not included in the calculation of $\mathrm{p}$ value for proportion of $\mathrm{HCP}$ among study groups.

to minimize further transmission were also neglected by the Brazilian testing policy ${ }^{6}$. The effect of this policy was documented in our data in which only one-third of patients with COVID-19-related symptoms had access to RT-PCR testing. Due to local testing policies, all HCP-CL performed tests when they were symptomatic, with no testing for the surveillance of asymptomatic individuals. The lower rate of RT-PCR testing, even in cases with symptoms suggestive of COVID-19 illustrates the limitation of these assays for the general, non-HCP, population.

The use of serology instead of direct viral RNA detection has proven inefficient to inform about the stage of disease and potential transmissibility, but the identification of antibodies is important in population-based studies to estimate the prevalence of COVID-19, providing a tool to assess the dynamics of the pandemic ${ }^{7}$.

Large population-based studies such as the one conducted by Hallal et al. ${ }^{8}$ estimated the prevalence of COVID-19 at the household level, but data in some specific groups remain limited or non-existent. People living with HIV or at risk of infection have many peculiarities that may impact COVID-19. In theory, individuals eligible for PrEP and PEP are engaged in behaviors that may disrespect the social distancing necessary to prevent SARS-CoV-2 transmission, which could be associated with a higher prevalence of COVID-19. In France, the risk of symptomatic COVID-19 appeared similarly in PLWH (15.6\%) and PrEP (14.8\%) users, in comparison with $19.1 \%$ in the general population ${ }^{9}$.

Garcia-Basteiro et al. ${ }^{10}$ found $9.3 \%$ (54/578) positivity in a survey carried out in Spain to estimate the seroprevalence of antibodies in HCP, in a large reference hospital. Data in Brazil are not available for PLWH or PrEP/PEP users, but when we compared our results to initial estimates for the area of our study based on the same RDT test, there was an estimated $2.9-4.9 \%$ positivity in the general population ${ }^{8}$, and $8.6 \%$ in $\mathrm{HCPs}^{11}$. These studies were conducted at an earlier time point, and the first study used the same RDT performed through a finger stick, which may be less sensitive than the detection of antibodies in plasma ${ }^{12}$.

A more recent serological survey carried out in the city of Sao Paulo, during January 2021, with the same RDT, but 
using plasma instead of whole blood, found a prevalence of $14.1 \%$, suggesting an increasing trend ${ }^{13}$. Interestingly, in this study, the prevalence among those that restricted contact with family members and friends was lower (11.4\%) than those that did not restrict contacts (28.9\%), which is similar to the results observed in our study. In another study in a large university clinic in the region, using the same RDT, $13.9 \%$ of 439 outpatients July 2020 tested positive ${ }^{14}$. An evaluation using the ECLIA assay on vulnerable populations of the metropolitan area, showed higher rates, $43.8 \%$ (95\% CI: $37.7-50 \%)^{15}$.

Our estimates for PrEP users were the lowest (12.5\%), but the confidence intervals (95\% CI: 2-23\%) overlapped other estimates. Together with estimates for PEP $(36 \%, 95 \%$ CI 21-54\%), our results do not support a higher prevalence associated with PrEP/PEP (23.7 95\% CI: 15-35\%) use or among PLWH $(31.3 \%, 95 \%$ CI: 8-41\%) when compared to HCP-CL $(21.4 \%, 95 \%$ CI: $8-41 \%)$ at this phase of the pandemic. The interpretation using ECLIA is similar, as is the combination of any positive test (RT-PCR or serological). A combination of results from different time points, may give higher estimates that may better reflect a previous COVID-19 status, as the interpretation of single time point serology, as in cross-sectional studies, have to take into account the waning of serological reactivity ${ }^{16,17}$.

In addition, the study documented an important disparity in the access to testing during this phase of the pandemic. With the reduction of cases and an expected fall of viral circulation in a fully vaccinated population, the emergence of variants among viremic, immunocompromised cases can become a source of new infections that may be minimized by an adequate COVID-19 monitoring and HIV suppression with antiretroviral therapy. Albeit small, this study provides baseline estimates of the pre-vaccination scenario. The observed prevalence is higher than other estimates for the metropolitan area of Sao Paulo and suggests the need for actions to prevent COVID-19, in addition to specific measures related to sexually transmitted infections in these individuals. The prevalence in the PrEP group may be a result of the limited number of cases studied and does not allow a proper interpretation. The study is limited by its small sample size and potential selection bias, however, the limited number of refusals among those invited to participate in the study makes this possibility less likely.

The study was carried out just before the vaccines were available in Brazil, and rates of performed serological tests should increase and reflect the sum of a natural antibody response to infection and the vaccination response. For many vaccine products, the combination of serological assays may provide a discrimination for the two sources of antibody production, as spike-based vaccines should not generate antibodies directed to the nucleoprotein antigen. However, in some parts of the world where inactivated virus vaccines were also used, this distinction may be less clear, and new assays that should ideally discriminate other coronaviruses, may be needed.

\section{AUTHORS' CONTRIBUTIONS}

All authors contributed to the study, commented on previous versions of the manuscript read, and approved the final manuscript. Conception and design, patient recruitment, data collection were performed and the manuscript was written by EMM. Patient recruitment, database registration, execution of rapid diagnostic tests were performed by IPO. Samples management and text preparation was performed by IBC. Serological tests and laboratory data collection were performed by CMA, MJC, VOS and GILL. Data registration and text review performed by FMM. Conception, statistical analysis, and manuscript review were performed by LFMB.

\section{CONFLICT OF INTERESTS}

The authors declare that there are no conflicts of interest.

\section{FUNDING}

The study was supported in part by a grant from CNPq/ MS-DIAHV Nº 24/2019-442776/2019-5.

\section{REFERENCES}

1. Seow J, Graham C, Merrick B, Acors S, Pickering S, Steel KJ, et al. Longitudinal observation and decline of neutralizing antibody responses in the three months following SARS-CoV-2 infection in humans. Nat Microbiol. 2020;5:1598-607.

2. World Health Organization. Global: Brazil. [cited 2021 Nov 8]. Available from: https://covid19.who.int/region/amro/country/ br/

3. Jangra S, Landers JJ, Rathnasinghe R, O’ Konek JJ, Janczak KW, Cascalho M, et al. A combination adjuvant for the induction of potent antiviral immune responses for a recombinant SARSCoV-2 protein vaccine. Front Immunol. 2021;12:729189.

4. Wang Y, Feng R, Xu J, Shi L, Feng H, Yang H. An updated meta-analysis on the association between HIV infection and COVID-19 mortality. AIDS. 2021;35:1875-8.

5. Centers for Disease Control and Prevention. Symptoms of COVID-19. [cited 2021 Nov 8]. Available from: https://www. cdc.gov/coronavirus/2019-ncov/symptoms-testing/symptoms. html 
6. Matsuda EM, Campos IB, Oliveira IP, Colpas DR, Carmo AM, Brígido LF. Field evaluation of COVID-19 antigen tests versus RT-PCR based detection: potential lower sensitivity compensated by immediate results, technical simplicity, and low cost. J Med Virol. 2021;93:4405-10.

7. Pellanda LC, Wendland EM, McBride AJ, Tovo-Rodrigues L, Ferreira MR, Dellagostin OD, et al. Sensitivity and specificity of a rapid test for assessment of exposure to SARS-CoV-2 in a community-based setting in Brazil. medRxiv. 2021 In Press.

8. Hallal PC, Hartwig FP, Horta BL, Silveira MF, Struchiner CJ, Vidaletti LP, et al. SARS-CoV-2 antibody prevalence in Brazil: results from two successive nationwide serological household surveys. Lancet Glob Health. 2020;8:e1390-8.

9. Charre C, Icard V, Pradat P, Brochier C, Lina B, Chidiac C, et al Coronavirus disease 2019 attack rate in HIV-infected patients and in preexposure prophylaxis users. AIDS. 2020;34:1765-70

10. Garcia-Basteiro AL, Moncunill G, Tortajada M, Vidal M, Guinovart C, Jiménez A, et al. Seroprevalence of antibodies against SARS-CoV-2 among health care workers in a large Spanish reference hospital. Nat Commun. 2020;11:3500.

11. Silva VO, Oliveira EL, Castejon MJ, Yamashiro R, Ahagon CM, López-Lopes GI, et al. Prevalence of antibodies against SARSCoV-2 in professionals of a public health laboratory at São Paulo, SP, Brazil. medRxiv 2021 In Press.
12. Satos VA, Rafael MM, Sabino EC, Duarte AJ. Sensitivity of the Wondfo One Step COVID-19 test using serum samples. Clinics (Sao Paulo). 2020;75:e2013.

13. São Paulo. COVID-19: ações 2021 [cited 2021 Nov 8]. Available from: http://www.capital.sp.gov.br/arquivos/pdf/2021/ coletiva_saude_14-01.pdf

14. Oliveira LM, Tiyo BT, Silva LT, Fonseca LA, Rocha RC, Santos VA, et al. Prevalence of anti-SARS-CoV-2 antibodies in outpatients of a large public university hospital in Sao Paulo, Brazil. Rev Inst Med Trop Sao Paulo. 2020;62:e91.

15. Miraglia JL, Monteiro CN, Romagnolo AG, Gomes RX, Mangueira CP, Rosseto-Welter EA, et al. A seroprevalence survey of anti-SARS-CoV-2 antibodies among individuals 18 years of age or older living in a vulnerable region of the city of São Paulo, Brazil. PLoS One. 2021;16:e0255412.

16. Van Caeseele P, Bailey D, Forgie SE, Dingle TC, Krajden M. SARS-CoV-2 (COVID-19) serology: implications for clinical practice, laboratory medicine and public health. CMAJ. 2020;192:E973-9

17. Townsend JP, Hassler HB, Wang Z, Miura S, Singh J, Kumar S, et al. The durability of immunity against reinfection by SARSCoV-2: a comparative evolutionary study. Lancet Microbe. 2021 In Press 